\title{
Teeth straightening pop-up launches at Westfield
}

Align Technology, Inc., the company that designs and manufactures Invisalign clear aligners and iTero intraoral scanners, has launched its first Invisalign Connect Pop-Up in London's Westfield shopping centre. The interactive pop-up is located in one of the city's busiest areas, with thousands of daily visitors - including prospective patients who may be interested in teeth straightening. By bringing the Invisalign brand directly to consumers in a digital and consumer-friendly environment, Align seeks to inform consumers about the benefits of well-aligned teeth, as well as to connect them with the trained Invisalign doctors of their choice who will determine if they are the right candidate for a treatment.

The first Invisalign Connect Pop-Up will bring the Invisalign brand closer to the consumers so they can:

- Browse, ask questions and learn about the benefits of teeth straightening with an Invisalign treatment

- Take the Smile Assessment or SmileView simulation of what their smile could look like after treatment

- Learn more about the cutting-edge technology behind Invisalign aligners

- Be connected to the Invisalign trained

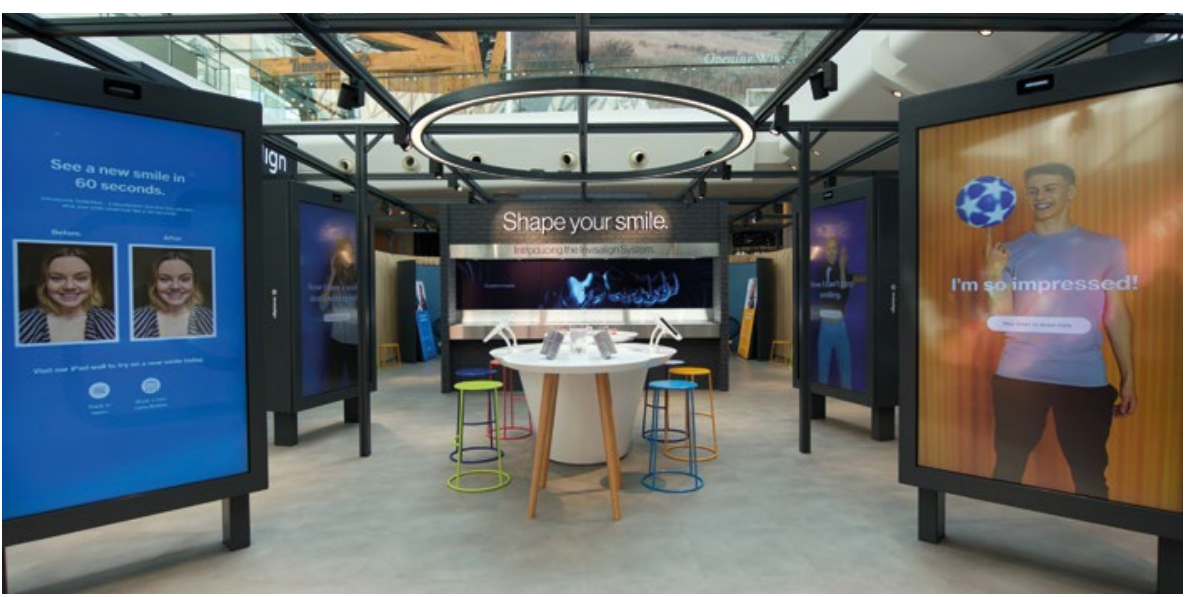

doctor of their choice to determine if they are a good candidate for an Invisalign treatment.

Invisalign staff present onsite will help the visitors navigate through the pop-up's digital consumer journey and answer questions related to teeth straightening. Invisalign trained staff are not clinicians or doctors. The consumers interested in a treatment will be referred directly to an Invisalign trained doctor of their choice to assess their suitability for the treatment.
The Invisalign system is the most technically advanced clear aligner system in the world and the most recognised brand in orthodontics. Globally, 6.8 million Invisalign patients have been treated with the Invisalign system, with more than 1 million patients in EMEA.

The Invisalign system is doctor-prescribed clear aligner treatment. Consumers in the UK interested in teeth straightening can visit an Invisalign trained doctor of their choice who will assess if the patient is applicable for clear aligner therapy.

\section{Longer lasting and more patient friendly}

Trycare is the UK Distributor for the complete Tokuyama range including award winning Sofreliner Tough, voted Top Denture Reline Material 2019 by the Dental Advisor.

Indicated for chairside relining of dentures, it is available as Sofreliner Tough Soft and Medium. Both provide

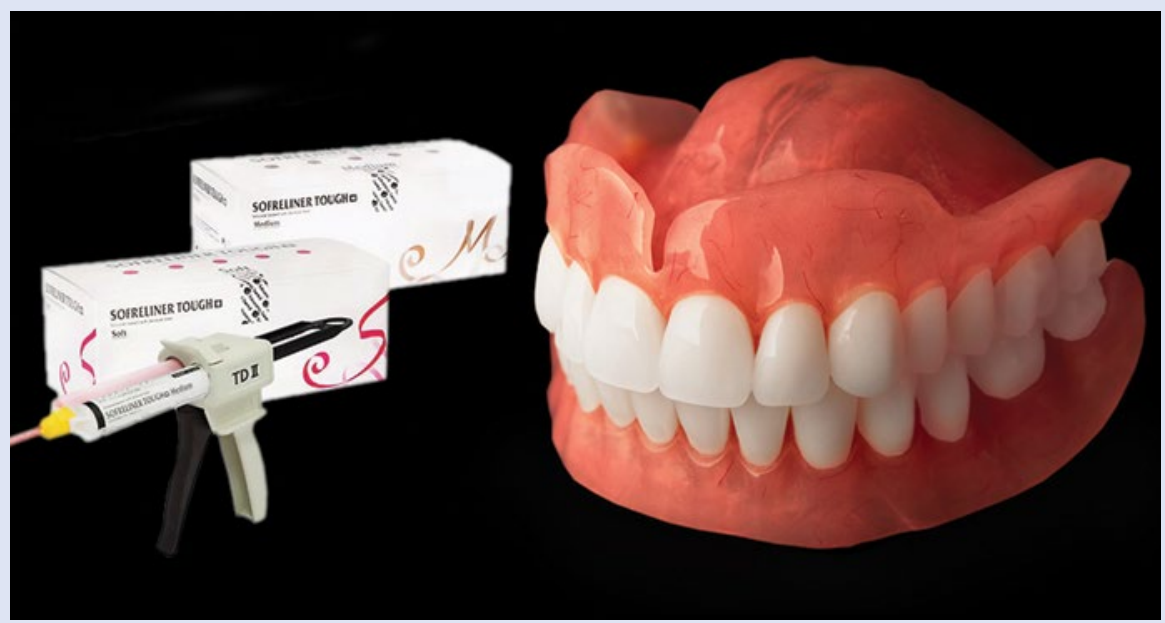

long-lasting relief from the pain of sharp alveolar ridges, mucous atrophy, major undercuts, maxillary or mandibular tori. They are also indicated for use in overdentures, improving suction and by first time denture wearers. Sofreliner Tough Soft is also indicated to ease tissue irritation, after surgery or tooth extraction and to aid implant osseointegration during the healing phase.

Sofreliner Tough Soft is a combination of soft reliner and tissue conditioner, whilst Sofreliner Tough Medium is an out and out soft reliner. Both provide long-lasting softness for maximum patient comfort. They also deliver superior tear strength, so they don't become worn and torn; have an extremely high stain resistance, so they maintain their appearance for longer; have a very strong adhesion to dentures, so they don't peel off around the margins; and maintain a lasting surface smoothness that alleviates contamination and the risk of odour build-up.

Automix dispensed via an XS mixing tip which provides $40 \%$ less waste than tips used by other manufacturers.

For more information about the complete Sofreliner Tough range, contact your local Trycare Representative, call 01274885544 or visit www.trycare.co.uk. 